\title{
Femtosecond laser writing of multifunctional optical waveguides in a Nd:YVO + KTP hybrid system
}

\author{
Ningning Dong, ${ }^{1}$ Yang Tan, ${ }^{1}$ Antonio Benayas, ${ }^{2}$ J. Vázquez de Aldana, ${ }^{3}$ Daniel Jaque,,${ }^{2, *}$ \\ Carolina Romero, ${ }^{3}$ Feng Chen, ${ }^{1,4,6}$ and Qingming Lu $^{5}$ \\ ${ }^{1}$ School of Physics, Shandong University, Jinan 250100, China \\ ${ }^{2}$ Fluorescence Imaging Group, Departamento de Física de Materiales, Facultad de Ciencias, \\ Universidad Autónoma de Madrid, Madrid 28049, Spain \\ ${ }^{3}$ Grupo de Óptica, Departamento Física Aplicada, Facultad Ciencias Físicas, Universidad de Salamanca, Salamanca 37008, Spain \\ ${ }^{4}$ State Key Laboratory of Crystal Materials, Shandong University, Jinan 250100, China \\ ${ }^{5}$ School of Chemistry and Chemical Engineering, Shandong University, Jinan 250100, China \\ ${ }^{6}$ e-mail: drfchen@sdu.edu.cn \\ ${ }^{*}$ Corresponding author: daniel.jaque@uam.es
}

Received January 5, 2011; accepted February 8, 2011;

posted February 16, 2011 (Doc. ID 140560); published March 11, 2011

\begin{abstract}
We report on the fabrication of optical channel waveguides supporting both visible and IR TE and TM confinement in a hybrid system composed of a $\mathrm{Nd}: \mathrm{YVO}_{4}$ laser gain medium glued to a potassium titanyl phosphate (KTP) nonlinear crystal. The microphotoluminescence and second harmonic confocal images of the fabricated waveguides have revealed that the laser and nonlinear properties of the constituent crystals have not been deteriorated because of the waveguide inscription. The resulting structures emerge as promising candidates for the development of multifrequency waveguide lasers. (c) 2011 Optical Society of America

OCIS codes: $140.3390,130.3120,140.0140,160.5690$.
\end{abstract}

Direct laser writing (DLW) with femtosecond pulses is nowadays regarded as one of the most versatile techniques for the fabrication of optical waveguides (WGs) in transparent materials. DLW of WGs is based on the refractive index changes (RICs) caused in a translating sample by tightly focused femtosecond laser pulses. These RICs appear after the complete relaxation of the multiphoton-induced transient plasma due to the creation of microstructural modifications at the focus and in its surroundings. Since the first demonstration by Davis et al. [1], DLW of optical WGs has been successfully applied in a great variety of systems ranging from glasses to transparent ceramics [1-12]. The properties of the resulting WGs have been found to be strongly determined by the writing conditions such as focusing conditions, polarization, pulse energy, pulse duration, repetition rate, and translation speed [4,5,9-13]. Nevertheless, the "writing window," defined by the different parameter ranges within which the induced RICs are large enough to create optical WGs, is usually a "broad window," and optical WGs can usually be fabricated by several combinations of the writing parameters. This would allow the DLW of optical WGs in different materials with exactly the same writing parameters. This possibility becomes highly interesting in hybrid optical systems, i.e., optical systems comprising two or more different materials, such as diffusion-bonded systems or composite elements made width glued crystals [13-15]. Of particular relevance is the combination of highly efficient IR laser elements (such as $\mathrm{Nd}: \mathrm{YVO}_{4}$ crystals) [ㅁ, $]$ ] with highly efficient frequency converter nonlinear crystals [such as potassium titanyl phosphate (KTP)] [16,17]. In this case the resulting hybrid system is capable of efficient self-frequency laser action in the visible (at $532 \mathrm{~nm}$ ) under IR (at $808 \mathrm{~nm}$ ) diode excitation of the $\mathrm{Nd}: \mathrm{YVO}_{4}$ $1064 \mathrm{~nm}$ laser transition. In this self-frequency-doubling (SFD) system, the overall IR-to-visible conversion effi- ciency could be improved with respect to bulk if both IR laser action and the subsequent SFD are achieved within a single optical WG. This improvement is based on the higher photon densities that can be achieved within WGs due to the strong two-dimensional confinement simultaneously leading to lower laser thresholds, larger laser slope efficiencies, and a more efficient $1064 \rightarrow$ $532 \mathrm{~nm}$ nonlinear frequency conversion in the KTP crystal. Despite their technological promise, no attempts to fabricate optical WGs in the Nd:YVO $\mathrm{YO}_{4}$ KTP SFD hybrid system have been reported up to now.

In this Letter we have fabricated optical WGs in a $\mathrm{Nd}: \mathrm{YVO}_{4}+$ KTP hybrid system by DLW under the "double-line" configuration. The polarized propagation properties of the resulting WGs have been analyzed in both the visible and IR spectral ranges. The potential use of the fabricated WGs as integrated SFD laser sources has been evaluated with the analysis of the confocal microphotoluminescence ( $\mu$-PL) and micro-secondharmonic $(\mu-\mathrm{SH})$ images of the $\mathrm{Nd}: \mathrm{YVO}_{4}$ and KTP end faces, respectively.

The Nd: $\mathrm{YVO}_{4}+$ KTP hybrid system used in this work comprised an $a$-cut $\mathrm{Nd}: \mathrm{YVO}_{4}$ crystal $\left(2\right.$ at. $\% \mathrm{Nd}^{3+}$ doping level, $4 \mathrm{~mm}$ in length) glued to a 4-mm-long KTP crystal cut along the $1064 \rightarrow 532 \mathrm{~nm}$ Type II SH direction. The dimensions of the hybrid system were $6 \mathrm{~mm} \times 1.5 \mathrm{~mm} \times$ $8 \mathrm{~mm}$, and it was designed for SFD laser action along the longest $(8 \mathrm{~mm})$ dimension. In addition to the two $6 \mathrm{~mm} \times 1.5 \mathrm{~mm}$ end faces, one of the $6 \mathrm{~mm} \times 8 \mathrm{~mm}$ faces was also polished to optical quality in order to allow DLW along the longest dimension.

Figure 1 shows a schematic drawing of the DLW process. In the fabrication procedure we used an amplified Ti:sapphire femtosecond laser operating at a wavelength of $800 \mathrm{~nm}$, with a repetition rate of $1 \mathrm{kHz}$ and a pulse duration of $100 \mathrm{fs}$. The laser beam was focused at a $50 \mu \mathrm{m}$ depth into the $\mathrm{Nd}: \mathrm{YVO}_{4}+\mathrm{KTP}$ hybrid system by 


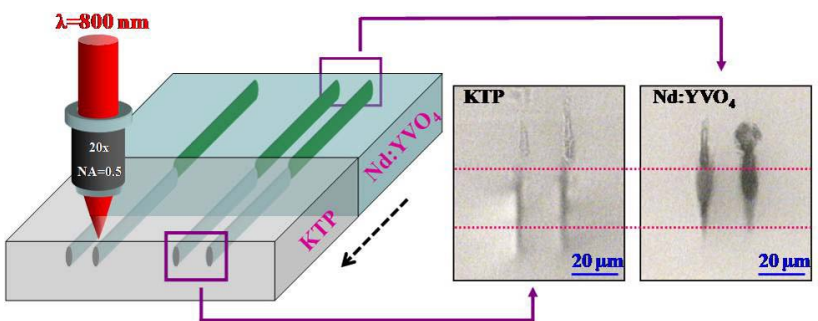

Fig. 1. (Color online) Schematic drawing of the experimental setup used for the fabrication of channel WGs in a Nd:YVO ${ }_{4}+$ KTP hybrid system. Dashed arrow indicates the translation direction of the hybrid sample. The optical transmission images of the WG as obtained from both the Nd: $\mathrm{YVO}_{4}$ and KTP end faces are also shown. Pictures correspond to the WG written with a pulse energy of $1.8 \mu \mathrm{J}$.

use of a $20 \times$ microscope objective (N.A. $=0.5$ ). This writing depth is 5 times less deep than that previously used in the fabrication of efficient laser WGs in single $\mathrm{Nd}: \mathrm{YVO}_{4}$ crystals by DLW $(250 \mu \mathrm{m})$ [6]. This reduced focusing depth was chosen in order to maximize the spatial overlap between the WGs fabricated in the $\mathrm{Nd}_{\mathrm{YVO}}$ and KTP crystals, since reduced focusing depths minimize the focus shift caused by the different refractive indices. The $\mathrm{Nd}: \mathrm{YVO}_{4}+\mathrm{KTP}$ hybrid system was translated along its longest dimension (orthogonal to the propagation direction of the femtosecond writing pulses) at a speed of $25 \mu \mathrm{m} / \mathrm{s}$. Based on the excellent results previously obtained in single $\mathrm{Nd} \mathrm{YVO}_{4}$ crystals [6], WGs were fabricated following the so-called "double-line" design. In this case WG formation requires the inscription of two parallel damage tracks separated by a reduced distance ( $20 \mu \mathrm{m}$ in our case). Under these experimental conditions, we fabricated continuous $\mathrm{Nd}: \mathrm{YVO}_{4}+\mathrm{KTP}$ WGs by using on-target pulse energies above $1 \mu \mathrm{J}$, with the best WGs obtained with $1.8 \mu \mathrm{J}$.

The cross-sectional optical transmission pictures of the WG fabricated with a pulse energy of $1.8 \mu \mathrm{J}$, as obtained from the Nd:YVO ${ }_{4}$ and KTP end faces, have been included in Fig. 1. As can be observed for these relatively large pulse energies, the length of the damaged zones is in excess of $30 \mu \mathrm{m}$. This makes possible a relevant special overlap between the features inscribed in the $\mathrm{Nd}: \mathrm{YVO}_{4}$ and KTP crystals, despite the slightly different focusing depths. This appreciable spatial overlap made possible complete light confinement, i.e., from the $\mathrm{Nd}: \mathrm{YVO}_{4}$ end face to the KTP end face. A detailed inspection of the cross-sectional views included in Fig. 1 reveals a larger focus shift in the KTP crystal than in the $\mathrm{YVO}_{4}$ one. Nevertheless, the larger refractive index of $\mathrm{YVO}_{4}$ would cause (only considering linear propagation of femtose-

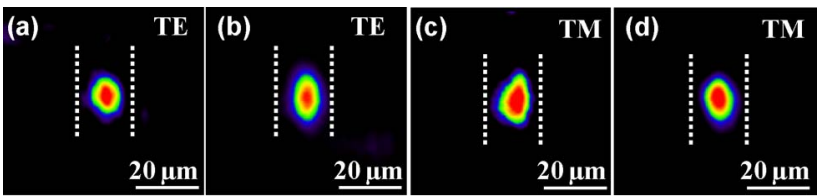

Fig. 2. (Color online) Near-field intensity distribution of (a) and (c) visible $(632.8 \mathrm{~nm})$ and (b) and (d) IR (1064 nm) WG modes as obtained for both TE and TM polarizations. Dashed lines indicate the position of the damage tracks. Scale bar is $20 \mu \mathrm{m}$. Data correspond to the WG fabricated with a pulse energy of $1.8 \mu \mathrm{J}$. cond pulses) a larger refraction-induced focus shift in the $\mathrm{Nd}: \mathrm{YVO}_{4}$ crystal (not observed experimentally). Thus, we attribute the different focus depths obtained to the different nonlinear propagation properties of femtosecond pulses in the two systems. Figure 2 shows both the TE and TM WG modes as obtained in the visible and IR spectral ranges. Waveguide modes were obtained by coupling into the WG either the visible beam generated from a He-Ne laser (at $632.8 \mathrm{~nm}$ ) or a fiber-coupled Nd:YAG laser operating at $1064 \mathrm{~nm}$. The WGs were capable of light confinement in both polarizations and in both the visible and IR ranges. This is an outstanding feature, since it is the first requirement that a WG should satisfy for efficient KTP-based Type II SFD (it implies the propagation of overlapping visible and IR beams with orthogonal polarizations).

The propagation losses of the hybrid WG were measured to be 3.9 and $4.6 \mathrm{~dB} / \mathrm{cm}$ for TE and TM polarizations at $632.8 \mathrm{~nm}$, respectively. Based on numerical simulations, the coupling loss between the two sections has been estimated to be $2.9 \mathrm{~dB}$. Assuming propagation losses of $1 \mathrm{~dB} / \mathrm{cm}$ for the $\mathrm{Nd}: \mathrm{YVO}_{4} \mathrm{WG}$ [6], we have estimated the propagation losses corresponding to the KTP WG segment ( 0.68 and $1.38 \mathrm{~dB} / \mathrm{cm}$ for TE and TM polarizations, respectively). In addition, the refractive index increment induced within the WG volume has been estimated, based on previous works, to be close to $1 \times 10^{-3}$ in both Nd: $\mathrm{YVO}_{4}$ and KTP WG segments [6,18].

The potential application of the fabricated structures as SFD WGs would also be determined by the preservation, at the WG volume, of the original (laser and nonlinear) properties of the hybrid system after the DLW process. In order to check this, we have measured the confocal $\mu$-PL and $\mu$-SH images of the Nd: $\mathrm{YVO}_{4}$ and KTP end faces, respectively. For this purpose we have used a fiber-coupled confocal microscope. The laser beam generated from a mode-locked Ti:sapphire laser ( $808 \mathrm{~nm}$ wavelength, $100 \mathrm{fs}$ pulse width and $80 \mathrm{MHz}$ repetition rate) was focused at the WG end faces using a $100 \times$ microscope objective $($ N.A. $=0.9)$. The backscattered radiation (PL or SH, depending on the end face under study) was collected with the same microscope objective and, after passing a set of filters and pinholes, it was coupled into an optical fiber connected to a high-resolution spectrograph. The Nd: $\mathrm{YVO}_{4}+\mathrm{KTP}$ sample was placed onto an $x y$ translation stage in such a way that it was possible to scan the $808 \mathrm{~nm}$ excitation spot over the WG cross section. The obtained images are shown in Fig. 3. As can be observed from the left image, the nonlinear response of the KTP crystals has not been modified in the WG's volume (i.e., between damage tracks). As a matter of fact, the SH response has been found to be modified only at the top of the damage tracks and in the core of them. The SH reduction observed at top apexes is attributed to the presence of surface ablation produced when the writing beam crosses the air/crystal interface. On the other hand, the relevant $\mathrm{SH}$ enhancement taking place at the damage zones has been attributed to an enhancement in the backreflection efficiency due to the presence of defects acting as scattering centers. The observed preservation of the nonlinear properties within the WG volume is in accordance with previous results related to the nonlinear properties of double-line WGs fabricated in nonlinear crystals 


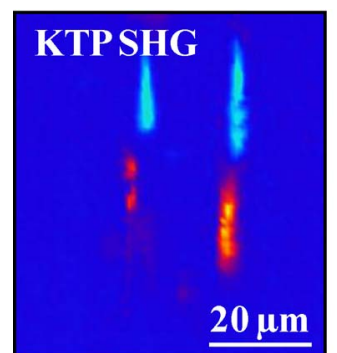

0.4

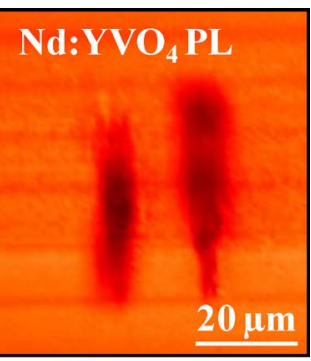

15
0.35
Fig. 3. (Color online) Left: Confocal $\mu$-SH cross-sectional image of a WG fabricated in the $\mathrm{Nd}: \mathrm{YVO}_{4}+\mathrm{KTP}$ hybrid system as obtained from the KTP end face. Right: Confocal $\mu$-PL crosssectional image of a WG fabricated in the Nd: $\mathrm{YVO}_{4}+\mathrm{KTP}$ hybrid system as obtained from the $\mathrm{Nd}: \mathrm{YVO}_{4}$ end face. Scale bar is $20 \mu \mathrm{m}$. Data correspond to the WG fabricated with a pulse energy of $1.8 \mu \mathrm{J}$.

$[\underline{19}, 20]$. Besides, the $\mu$-PL image shown in Fig. 3 (right) proves that the $\mathrm{Nd}^{3+}$ fluorescence intensity of the $1064 \mathrm{~nm}$ laser line is well preserved within the WG volume, being reduced only at the damage tracks due to defect-assisted fluorescence quenching. The images of Fig. 3 indicate that both the nonlinear and laser gain properties of the $\mathrm{Nd}: \mathrm{YVO}_{4}+\mathrm{KTP}$ hybrid system have not been deteriorated as a consequence of the WG fabrication procedure.

In summary, an adequate choice of parameters has enabled our fabrication of buried channel optical WGs in a $\mathrm{Nd}: \mathrm{YVO}_{4}+\mathrm{KTP}$ hybrid system by femtosecond laser writing. The resulting WGs were found to support polarization-independent light confinement in both the visible and IR bands. Confocal $\mu$-PL and $\mu$-SH images have revealed that both the fluorescence properties of $\mathrm{Nd}: \mathrm{YVO}_{4}$ and the nonlinear response of the KTP have been well preserved within the WG volume, which suggests the formed structures are promising candidates for the near-future development of efficient SFD laser WGs operating in the visible. Indeed, preliminary experiments have already shown this fact; we have obtained up to $50 \mu \mathrm{W}$ at $532 \mathrm{~nm}$ when the Nd:YVO ${ }_{4}$ WG segment was optically excited at a wavelength of $808 \mathrm{~nm}$ with a launched power of $600 \mathrm{~mW}$. Several attempts to improve this preliminary IR-to-green conversion efficiency will be made in the near future.

This work is supported by the National Natural Science Foundation of China (NSFC) (No. 10925524), the Spanish Ministerio de Ciencia y Tecnología (MAT 2010-16161, MAT2009-06580-E/MAT, FIS2009-09522, and CSD2007-00013), the Consejería de Educación de la Comunidad de Madrid, Universidad Autónoma de Madrid (UAM-CAM) (CCG08-UAM/MAT-4434 and PHAMA P2009 /MAT1756).

\section{References}

1. K. M. Davis, K. Miura, N. Sugimoto, and K. Hirao, Opt. Express 21, 1729 (1996).

2. K. Miura, J. R. Qiu, H. Inouye, T. Mitsuyu, and K. Hirao, Appl. Phys. Lett. 71, 3329 (1997).

3. M. Hughes, W. Yang, and D. Hewak, Appl. Phys. Lett. 90, 131113 (2007).

4. S. Nolte, M. Will, J. Burghoff, and A. Tuennermann, Appl. Phys. A 77, 109 (2003).

5. M. Ams, G. D. Marshall, and M. J. Withford, Opt. Express 14, 13158 (2006).

6. W. F. Silva, C. Jacinto, A. Benayas, J. R. Vazquez de Aldana, G. A. Torchia, F. Chen, Y. Tan, and D. Jaque, Opt. Lett. 35, 916 (2010).

7. S. J. Beecher, R. R. Thomson, N. D. Psaila, Z. Sun, T. Hasan, A. G. Rozhin, A. C. Ferrari, and A. K. Kar, Appl. Phys. Lett. 97, 111114 (2010).

8. R. Osellame, M. Lobino, N. Chiodo, M. Marangoni, G. Cerullo, R. Ramponi, H. T. Bookey, R. R. Thomson, N. D. Psaila, and A. K. Kar, Appl. Phys. Lett. 90, 241107 (2007).

9. A. H. Nejadmalayeri and P. R. Herman, Opt. Express 15, 10842 (2007).

10. A. Ródenas, G. A. Torchia, G. Lifante, E. Cantelar, J. Lamela, F. Jaque, L. Roso, and D. Jaque, Appl. Phys. B 95, 85 (2009).

11. G. A. Torchia, P. F. Meilán, A. Rodenas, D. Jaque, C. Mendez, and L. Roso, Opt. Express 15, 13266 (2007).

12. A. Benayas, W. F. Silva, C. Jacinto, E. Cantelar, J. Lamela, F. Jaque, J. R. Vázquez de Aldana, G. A. Torchia, L. Roso, A. A. Kaminskii, and D. Jaque, Opt. Lett. 35, 330 (2010).

13. J. Z. Sotor, A. J. Antończak, and K. M. Abramski, Optoelectron. Rev. 18, 75 (2010).

14. C. Jung, B. A. Yu, I. S. Kim, Y. L. Lee, N. E. Yu, and D. K. Ko, Opt. Express 17, 19611 (2009).

15. C. Jung, B. A. Yu, K. Lee, Y. L. Lee, N. E. Yu, D. K. Ko, and J. Lee, Appl. Phys. Expr. 1, 062005 (2008).

16. T. A. Driscoll, H. J. Hoffman, R. E. Stone, and P. E. Perkins, J. Opt. Soc. Am. B 3, 683 (1986).

17. K. Tei, M. Kato, F. Matsuoka, Y. Niwa, Y. Maruyama, T. Matoba, and T. Arisawa, Jpn. J. Appl. Phys. 38, L35 (1999).

18. J. Siebenmorgen, K. Petermann, G. Huber, K. Rademaker, S. Nolte, and A. Tünnermann., Appl. Phys. B 97, 251 (2009).

19. Y. Liao, J. Xu, Y. Cheng, Z. Zhou, F. He, H. Sun, J. Song, X. Wang, X. Xinshun, S. Zhizhan, K. Sugioka, and K. Midorikawa, Opt. Lett. 33, 2281 (2008).

20. J. Thomas, M. Heinrich, P. Zeil, V. Hilbert, K. Rademaker, R. Riedel, S. Ringleb, C. Dubs, J.-P. Ruske, S. Nolte, and A. Tünnermann, Phys. Stat. Sol. A 208, 276 (2011). 